\title{
ESTRATÉGIAS DAS GRANDES EMPRESAS FARMACÊUTICAS NACIONAIS NA INTERAÇÃO EMPRESA-ICT ${ }^{1}$
}

Julia Paranhos, Professora Adjunta; Grupo de Economia da Inovação do Instituto de Economia/Universidade Federal do Rio de Janeiro (juliaparanhos@ie.ufrj.br).

Fernanda Steiner Perin, doutoranda em Economia da Indústria e da Tecnologia pelo IE/UFRJ (fernanda.steinerperin@gmail.com).

Eduardo Mercadante, mestrando em Políticas Públicas, Estratégias e Desenvolvimento pelo IE/UFRJ (edu.mercadante@ufrj.br).

Caroline Soares, graduanda em Ciências Econômicas no IE/UFRJ (caroline.soares6@hotmail.com)

\section{RESUMO}

O objetivo do trabalho é analisar as estratégias e as formas organizacionais empregadas pelas grandes empresas farmacêuticas nacionais (GEFNs) nas parcerias com Instituições Científicas e Tecnológicas (ICTs) para a geração de inovação. No setor farmacêutico, indústria baseada em ciência, a fonte de novos conhecimentos se encontra, frequentemente, externa ao ambiente da empresa. Deste modo, a busca pela inovação requer uma definição estratégica por parte da empresa. A pesquisa foi realizada com o método de estudo de caso, com os dados da Pesquisa de Inovação (Pintec) 2014 sobre os esforços inovativos das GEFNs e por meio de uma pesquisa de campo com seis GEFNs. Os dados mais recentes da pesquisa de inovação no Brasil mostram mudanças positivas nos esforços inovativos das GEFNs, envolvendo a maior aproximação com ICTs. Os resultados também mostram que as GEFNs possuem estruturas inovativas diferenciadas e estão empregando efetivamente estratégias para as parcerias com ICTs, por meio da criação de departamentos de inovação radical, do estabelecimento de comitês científicos internos e da internacionalização da pesquisa e desenvolvimento.

Palavras-chave: Estratégias empresariais, interação universidade-empresa, setor farmacêutico, Brasil.

\section{ABSTRACT}

The aim of this paper is to analyse the strategies and organizational forms used by big national pharmaceutical companies (BNPCs) in interaction with Scientific and Technological Institutions (STI) for the development of innovation. In the pharmaceutical sector, a sciencebased industry, the source of new knowledge is often outside the company environment. Thus, the search for innovation requires a strategic decision by the company. The research uses the case study method, with data from the Innovation Survey (Pintec, in Portuguese) from 2014 about the innovative efforts of BNPCs and a field work on six BNPCs. The most

\footnotetext{
1 Pesquisa financiada pelo edital CNPq 41/2013. Os autores agradecem à coordenação da Pintec/IBGE pela disponibilização dos dados por meio da tabulação especial.
} 
recent data on innovation in Brazil show positive changes in the innovative efforts of BNPCs, involving greater interaction with STI. The results also show that the BNPCs have differentiated innovative structures and are effectively employing strategies for partnerships with STIs, through the creation of departments of radical innovation, the establishment of internal scientific committees and the internationalization of research and development.

Key words: Company strategies, Industry-University Interaction, Pharmaceutical sector, Brazil.

Área 3: Estratégias empresariais, financiamento e governança corporativa.

3.4 Estratégias e gestão da C\&T\&I

JEL: O30, 032.

\section{Introdução}

O estudo do papel da universidade no processo inovativo das empresas tem recebido um crescente destaque na literatura brasileira nos últimos anos (DEL-VECCHIO; BRITTO; OLIVEIRA, 2013; CHAVES et al., 2012; PARANHOS, 2012; SUZIGAN; ALBUQUERQUE, 2011; RAPINI et al., 2009). Tal interesse tem sido motivado pelo reconhecimento das instituições científicas e tecnológicas (ICTs) como um complemento importante na geração de inovação das empresas. Neste sentido, este estudo contribui para melhorar a compreensão do tema, na medida em que analisa quais são as estratégias e as formas organizacionais empregadas pelas grandes empresas farmacêuticas nacionais (GEFNs) nas parcerias com ICTs para a geração de inovação.

A inovação é um processo social, uma vez que envolve a participação de diferentes atores, e não possui uma fonte geograficamente localizada; logo, necessita de uma definição estratégia por parte da empresa para a sua geração. A empresa que se engaja em inovação incorre em duas decisões inter-relacionadas. A primeira delas se refere à estratégia, em que a empresa precisa balancear entre a busca por novas oportunidades e a exploração de suas capacidades existentes. A segunda decisão é como melhor alocar os recursos para pesquisa e desenvolvimento (P\&D) entre atividades internas e alianças externas (BERCOVITZ; FELDMAN, 2007).

A capacidade de explorar fontes externas de conhecimento tornou-se cada vez mais importante para as empresas que buscam obter vantagem competitiva por meio da inovação. Os acordos colaborativos são um meio pelo qual as empresas obtêm acesso ao ambiente de conhecimento externo (ARORA; GAMBARDELLA, 1990).

O processo de decisão por parte da empresa quanto à sua estratégia de colaboração perante o desenvolvimento de uma inovação tecnológica consiste em identificar a melhor forma de obter vantagem no desempenho de uma atividade. Schilling (2006) argumenta que as empresas podem desenvolver a inovação sozinhas, tendo, assim, maior domínio sobre o processo integral e sobre a apropriação da inovação. Porém, as empresas podem também desenvolver a inovação em colaboração, sendo, então, compartilhado o domínio sobre o processo e menor a capacidade de apropriação. No entanto, nesses casos, novas capacidades podem ser absorvidas e desenvolvidas a partir da interação, gerando benefícios à empresa.

Entre as vantagens que motivam uma empresa a engajar-se em um empreendimento conjunto, destaca-se a aquisição de recursos ou capacidades necessárias para o desenvolvimento de um projeto por meio da parceria mais rapidamente que no desenvolvimento individual. Com a 
colaboração, a empresa ganha flexibilidade na produção, pois compartilha o comprometimento com o projeto, além de a parceria poder ser uma fonte de aprendizado, cujo contato aproximado facilita a transferência de conhecimento. Os custos e os riscos do projeto também são divididos, sendo que o interesse pela colaboração aumenta em proporção direta ao valor do projeto e à incerteza sobre os resultados (SCHILLING, 2006).

As empresas entram em diferentes tipos de atividades colaborativas dependendo do tipo de conhecimento que procuram adquirir. De acordo com Hess e Rothaermel (2011), as empresas podem fazer alianças com o propósito de procurar novas oportunidades, tendo como foco principal a geração de novos conhecimentos básicos, ou mesmo a geração de conhecimento mais aplicado, para explorar uma capacidade existente. Hottenrott e Lopes-Bento (2012) evidenciaram que as colaborações do primeiro tipo, em que as partes empreendem em um projeto de $\mathrm{P} \& \mathrm{D}$ conjunto, têm maiores efeitos sobre a produtividade da $\mathrm{P} \& \mathrm{D}$ e a produção de inovação. Na mesma linha, Teece (2007) afirma que a criação de novas capacitações, no lugar da mera exploração das já existentes, leva à conquista de capacidades dinâmicas, permitindo a adaptação da empresa ao ambiente competitivo em transformação e renovando suas competências com respostas inovadoras. Independentemente do tipo, a P\&D colaborativa é um instrumento usado pelas empresas para adquirir novas habilidades e obter know-how especializado, de modo que pode ser vista como uma resposta à mudança no ambiente de conhecimento, permitindo que as empresas lidem com os desafios tecnológicos.

A interação universidade-empresa difere das alianças interempresas, uma vez que a pesquisa acadêmica está situada em um ambiente que incentiva a liberdade de explorar novas ideias e a troca de conhecimento científico. Assim, o escopo da pesquisa associada às colaborações com ICTs é geralmente menos definido e mais distante dos objetivos comerciais. Ao traduzir a pesquisa acadêmica em aplicações comerciais, as empresas devem possuir a capacidade de acessar e aplicar o conhecimento que é novo, incluindo componentes explícitos e tácitos (ARORA; GAMBARDELLA, 1990).

A universidade, enquanto parceira inovativa, está mais isolada das demandas competitivas e, portanto, pode perseguir uma pesquisa mais ampla e, subsequentemente, oferecer um conjunto mais exclusivo de know-how. O novo conhecimento gerado pela pesquisa universitária pode fornecer avanços importantes com potencial comercial de longo prazo (ROSENBERG; NELSON, 1994). Além disso, Belderbos et al. (2004) identificaram que as interações entre empresas e ICTs estimulam a cooperação de outros tipos de parceria, pois o conhecimento obtido nas ICTs tem uma natureza mais genérica, melhorando as oportunidades tecnológicas e as estratégias de cooperação em P\&D da empresa.

As empresas não são capazes de se apropriar totalmente do valor do conhecimento criado por meio de seus investimentos em $\mathrm{P} \& \mathrm{D}$, e a decisão de participar de parcerias de $\mathrm{P} \& \mathrm{D}$ aumenta esse desafio. Embora o esforço de pesquisa colaborativa possa aumentar o potencial de descoberta, também aumenta o potencial de perda de controle sobre a propriedade intelectual gerada $^{2}$. Por esse motivo, são estabelecidos contratos que definem a participação de cada parte na divisão da propriedade intelectual (BERCOVITZ; FELDMAN, 2007).

É importante, porém, a empresa encontrar um equilíbrio entre os investimentos em P\&D interna e externa. Estudos (COHEN; NELSON; WALSH, 2002; VEUGELERS; CASSIMAN, 2005; BERCOVITZ; FELDAMN, 2007) mostram que as empresas com mais inovações são

\footnotetext{
${ }^{2}$ A possibilidade de perda da propriedade total do conhecimento está presente em toda e qualquer estratégia de parceria. No entanto, dada as vantagens das parcerias, as empresas buscam estabelecer formas de evitar o oportunismo de seus parceiros (SCHILLING, 2006).
} 
aquelas que equilibram investimentos em $\mathrm{P} \& \mathrm{D}$ interna e com parceiros. O investimento da empresa em P\&D interna é importante tanto para a criação de conhecimento novo para gerar inovações, quanto para ampliar a capacidade da empresa de absorver os conhecimentos externos. Assim, a P\&D interna cria capacidade de absorção, que possibilita o melhor aproveitamento das alianças inovativas com parceiros externos. Nas parcerias de desenvolvimento conjunto, inclusive com universidades, a necessidade de investir na geração de capacidade de absorção é fundamental, pois permite que ela reconheça o valor do conhecimento a ser assimilado e explorado (COHEN; LEVINTHAL, 1990).

A P\&D colaborativa entre empresas e ICTs é mais recorrente nos setores baseados em ciência, como o farmacêutico (PAVITT, 1984). Especificamente em indústrias intensivas em tecnologia, a fonte de novos conhecimentos se encontra, frequentemente, externa ao ambiente da empresa. A crescente complexidade e multidisciplinaridade da natureza do processo de inovação pressiona as empresas a acessar o conhecimento externo para apoiar suas atividades produtivas e inovativas, tanto a montante $(\mathrm{P} \& \mathrm{D})$, quanto a jusante (produção e marketing) da cadeia de valor. Desse modo, o desempenho inovador da empresa é afetado por sua capacidade de criação e gestão de interações com outras organizações (HESS; ROTHAERMEL, 2011).

Teece (1986) destacou a importância dos ativos complementares ao processo de desenvolvimento de inovações, os quais dão vantagens às grandes empresas para desenvolvimento da inovação, para chegar com a inovação no mercado, assim como, para apropriação da inovação. No caso do setor farmacêutico especificamente, a existência de ativos complementares é extremamente importante devido ao longo, custoso e complexo processo de desenvolvimento de novos medicamentos ${ }^{3}$ (Pimentel, 2010).

No ambiente internacional, as empresas farmacêuticas têm um longo histórico de parcerias com ICTs, sendo responsáveis por importantes avanços na área, tal como a biotecnologia (PISANO, 1991; DODGSON, 1993). Já no Brasil, o setor farmacêutico foi criado a partir da dependência externa com baixo desenvolvimento tecnológico nacional. Desde o final da década de 1990, mudanças no arcabouço institucional tentam reverter esse quadro, em que as empresas passaram a contar com políticas de apoio à produção local, à $P \& D$ e à inserção externa (HASENCLEVER et al., 2016).

A promulgação da Lei de Propriedade Industrial (LPI), Lei nº 9.279, em 1996, foi importante para trazer segurança jurídica às parcerias nacionais e para a abertura comercial da indústria farmacêutica brasileira, posto que nenhuma patente farmacêutica era concedida desde 1969, como estratégia de estímulo à capacitação tecnológica e industrial, com atenção à produção local e buscando reduzir os preços dos medicamentos. No entanto, essa medida se insere em um contexto de pouca atenção à política industrial, gerando um resultado negativo para a competitividade nacional frente às multinacionais. Como tentativa de introduzir alternativas para o desenvolvimento das empresas farmacêuticas nacionais (EFNs), foram criadas a Agência Nacional de Vigilância Sanitária (Anvisa), Lei no 9.782/1999, e a Lei dos Genéricos, Lei $n^{\circ}$ 9.787/1999 (DELGADO, 2015; CHAVES; HASENCLEVER; OLIVEIRA, 2016; PARANHOS; MERCADANTE, 2017).

Antes da LPI, era possível para as EFNs o investimento em engenharia reversa e o lançamento de similares aos medicamentos de referência patenteados, produzidos pelas multinacionais. Uma vez que a LPI proibiu essa estratégia, ao reintroduzir o patenteamento farmacêutico, as alternativas produziram incentivos para que as EFNs construíssem

\footnotetext{
${ }^{3}$ Para maiores detalhes ver Radaelli (2008).
} 
capacidades tecnológicas para atender aos requisitos de atuar no segmento de genéricos ${ }^{4}$. Além disso, o elevado grau de exigência da Anvisa pelos critérios de boas práticas laboratoriais e de produção ${ }^{5}$, ajudou a tornar o produto brasileiro atrativo internacionalmente. Por outro lado, muitas empresas de capital nacional encerraram suas operações, uma vez que não conseguiram desenvolver capacidades tecnológicas suficientes para atender às novas exigências da legislação (STRUCKER; CYTRYNOWICZ, 2007).

Em 2003, a implantação da Política Industrial, Tecnológica e de Comércio Exterior (PITCE), estabeleceu o relacionamento entre empresas e ICTs como estratégico para promoção da inovação, sendo comtemplado nas diversas ações governamentais seguintes: Fundos Setoriais de Ciência e Tecnologia (C\&T), Lei de Inovação ( $\left.\mathrm{n}^{\circ} 10.973 / 2004\right)$, Lei do Bem ( ${ }^{\circ}$ 11.196/2005) e o Marco de Ciência, Tecnologia e Inovação (Marco de CTI, nº 13.243/2016). O setor farmacêutico também foi destacado nas duas políticas industrias seguintes - Política de Desenvolvimento Produtivo (2008) e Plano Brasil Maior (2011) - e a promoção da interação empresa-ICT nas políticas de ciência, tecnologia e inovação - Programa de Aceleração do Crescimento de Ciência e Tecnologia (2007) e Estratégia Nacional de Ciência, Tecnologia e Inovação (2016-2019) ${ }^{6}$.

A atual configuração do mercado farmacêutico brasileiro é dada por empresas multinacionais, que fornecem medicamentos inovadores, e por empresas nacionais, que tem baixa capacidade inovativa com foco na fabricação de produtos com baixo valor agregado (CGEE, 2017). No entanto, os medicamentos inovadores são desenvolvidos nas matrizes ou centros de pesquisas das empresas multinacionais, onde realizam as atividades de P\&D e parcerias com ICTs, ficando apenas a parte de comercialização no Brasil (PARANHOS, 2012). Não obstante, a consolidação recente da indústria farmacêutica brasileira mediante as políticas e instrumentos de apoio levou ao redirecionamento das estratégias inovativas de algumas empresas nacionais, inserindo-se em inovação radical e buscando o mercado internacional como forma de aprimoramento de suas capacidades tecnológicas. Essa orientação reflete-se na interação com ICTs, em que são observadas a formação de diferentes tipos de parcerias.

$\mathrm{O}$ crescimento e destaque das grandes empresas farmacêuticas nacionais, potencialmente como resultado da política industrial e de inovação implementada, foi retratado em Paranhos, Mercadante e Hasenclever (2016). Uma análise global das parcerias empresa-ICT no setor farmacêutico foi realizada em Paranhos et al. (2018), evidenciando que as parcerias ainda são pontuais e focadas na prestação de serviços, consequência do perfil inovativo das empresas e morosidade dos processos nas ICTs. O objetivo neste artigo é dar continuidade a estas pesquisas e atualizar os dados das estratégias e formas de cooperação das grandes empresas farmacêuticas nacionais com ICTs. Para responder à pergunta de como e por que as GEFNs interagem com ICTs para gerar inovação, é realizada análise da Tabulação Especial da Pesquisa de Inovação do Instituto Brasileiro de Geografia Estatística (Pintec/IBGE) de 2014, referente às grandes empresas farmacêuticas nacionais (GEFNs), para criar um panorama inovativo e estratégias de setor. Adicionalmente, utiliza-se o método de análise de conteúdo de entrevistas realizadas em uma pesquisa de campo com seis grandes empresas

\footnotetext{
${ }^{4} \mathrm{Na}$ Lei 9.787/1999 foram exigidos os requisitos de biodisponibilidade, velocidade e extensão de absorção de um princípio ativo em uma forma de dosagem e de bioequivalência, equivalência farmacêutica entre produtos apresentados sob a mesma forma farmacêutica, contendo idêntica composição qualitativa e quantitativa de princípios ativos, e que tenham comparável biodisponibilidade.

${ }^{5}$ Certificados de Boas Práticas de Fabricação (CBPF) e de Boas Práticas de Armazenamento e Distribuição (CBPAD).

6 Informações mais detalhadas sobre as políticas direcionadas ao setor farmacêutico são encontradas em Hasenclever et al. (2016).
} 
farmacêuticas nacionais entre 2016 e 2017, para atualização e aprofundamento das características e estratégias das parcerias.

O artigo está dividido em três seções, além desta Introdução. Na primeira seção, é apresentada a metodologia. $\mathrm{Na}$ segunda seção, são apresentados os resultados da análise dos dados secundários - Pintec 2014 - e dos dados primários - pesquisa de campo com entrevistas com seis GEFN. Na terceira seção, é apresentada discussão e conclusão à luz da literatura de estudos realizados sobre o tema.

\section{Procedimentos metodológicos}

A interação universidade-empresa tem sido estudada no Brasil quanto a características como seus impactos inovativos, tipos de financiamento e formas de absorção (RAPINI; OLIVEIRA; CALIARI, 2016; TEIXEIRA et al., 2016; LEMOS; CARIO, 2015; CASTRO; TEIXEIRA; LIMA, 2014), destacando o papel das parcerias com empresas farmacêticas brasileiras (DEL-VECCHIO; BRITTO; OLIVEIRA, 2013; CHAVES et al., 2012; SUZIGAN; ALBUQUERQUE, 2011; RAPINI et al., 2009). Na literatura internacional, também existem estudos que tratam das colaborações de empresas farmacêuticas, de variadas nacionalidades, com ICTs (FILIERI et al., 2014; TRIULZI; PYKA; SCHOLZ, 2014; SOH; SUBRAMANIAN, 2014; BLAKESLEE, 2012; TRALAU-STEWART et al., 2009). Sendo assim, este estudo avança nesse tema de investigação, uma vez que especifica o tema de análise para as formas de parcerias e as estratégias das empresas empregadas na interação com ICT, utilizando fontes de dados primárias e secundárias.

Para alcançar o objetivo proposto, esta pesquisa foi realizada a partir do método de estudo de caso, pois busca compreender um fenômeno contemporâneo com alto grau de complexidade. O método é adequado para o estudo da interação empresa-ICT, uma vez que permite esclarecer os vínculos causais e a descrição de influências em que não há um conjunto claro de resultados (YIN, 2015; MARCONI; LAKATOS, 2016).

As técnicas empregadas foram revisão de literatura, análise quantitativa de estatística descritiva e pesquisa de campo. O estudo tem, portanto, um caráter exploratório e utiliza dados quantitativos e qualitativos. Por meio da revisão de literatura buscou-se aprimorar os conhecimentos sobre o problema de pesquisa e contrastar os resultados da pesquisa de campo com aqueles encontrados em pesquisas semelhantes.

A caracterização geral das GEFNs foi realizada a partir de dados secundários contidos em Tabulação Especial da Pintec 2014, produzida e fornecida pelo IBGE. A Pintec é uma pesquisa amostral que analisa diversas variáveis relacionadas ao esforço e desempenho inovativo de empresas no último triênio, baseada no Manual de Oslo da Organização para a Cooperação e Desenvolvimento Económico ou Econômico (OCDE). Como critérios qualificadores, foram adotados: a) para grande porte, o fato de a empresa ter pelo menos 500 pessoas ocupadas; b) para setor farmacêutico, o enquadramento na Classificação Nacional de Atividades Econômicas (CNAE) 21, Fabricação de produtos farmoquímicos e farmacêuticos; e c) para nacionais, o fato de o capital controlador da empresa ser nacional.

Por sua vez, a pesquisa de campo foi realizada, na sua maioria, presencialmente com empresas farmacêuticas nacionais por meio de entrevistas semiestruturadas e focalizadas. A pesquisa de campo ocorreu em seis etapas: a) determinação do objetivo; b) seleção dos entrevistados, c) elaboração de dossiês sobre as empresas; d) elaboração do questionário; e) 
estabelecimento de contato inicial e realização das entrevistas; f) transcrição, consolidação e análise das entrevistas. A amostra de empresas da pesquisa de campo que é analisada neste artigo consiste em seis grandes empresas farmacêuticas nacionais. A seleção das empresas foi feita de forma intencional para identificação de empresas com parcerias com ICT. Os temas abordados nas entrevistas se referem às atividades de $P \& D$ das grandes empresas farmacêuticas nacionais realizadas em parcerias com ICTs. Para preservar o anonimato do entrevistado, a identificação é feita por um código, que indica a ordem da entrevista entre todas as entrevistas realizadas com empresas farmacêuticas nacionais e o ano.

\section{A interação empresa-ICT no Brasil}

O estudo do relacionamento empresa-ICT no setor farmacêutico brasileiro tem sido aprofundado há alguns anos dada a importância que o setor ganhou na política industrial e de inovação nas duas últimas décadas. É possível destacar os instrumentos de apoio a essas parcerias e a relevância mostrada na literatura internacional dessa interação para um setor baseado em ciência, como o setor farmacêutico, conforme apresentado na Introdução. Nesta seção, são apresentados os dados secundários e primários sobre o panorama destas parcerias entre GEFNs e ICTs. A primeira subseção apresenta os dados secundários da tabulação especial para grandes empresas farmacêuticas de capital controlador nacional da Pintec 2014/IBGE, que tem o objetivo de apresentar o panorama do setor entre 2012 e 2014. A segunda subseção apresenta os dados primários da pesquisa de campo com seis GEFNs realizada entre 2016 e 2017, com objetivo de aprofundar e atualizar a apresentação do cenário das parcerias GEFN-ICT.

\subsection{Cenário das grandes empresas farmacêuticas na Pintec 2014}

A Pintec/IBGE 2014 mostra que as GEFNs ${ }^{7}$ inovam sozinhas, principalmente no caso da inovação de produto (cerca de $81,5 \%$ das GEFNs na edição de 2014), conforme já foi identificado nas Pintec 2008 e 2011 (PARANHOS; MERCADANTE; HASENCLEVER, 2016). Nas inovações de processo, outras empresas ou institutos ganham maior relevância na responsabilidade de desenvolvimento das inovações, sendo importantes para cerca de 45,8\% das GEFNs na Pintec 2014. A aquisição de P\&D externa e aquisição de outros conhecimentos externos foram atividades realizadas por $49 \%$ e $30 \%$ das GEFNs inovadoras entre 2012 e 2014 , ainda que somente $35 \%$ e $26 \%$, respectivamente, tenham identificado tais atividades como de alta importância.

Ainda que muitas realizem as atividades, os montantes investidos nas mesmas são bastante baixos, o que provavelmente reforça o fato de nem todas as empresas que realizam a atividade a identificarem como de alta importância. As GEFNs investiram 0,9\% das suas receitas líquidas de vendas (RLV) na aquisição de P\&D externa ${ }^{8}$ entre 2012 e 2014; em contraponto, os dispêndios em $\mathrm{P} \& \mathrm{D}$ interna foram $4,9 \%$ da $\mathrm{RLV}^{9}$. Vale notar, porém, que o financiamento

\footnotetext{
${ }^{7}$ A amostra de GEFNs inovadoras da Pintec de 2014 é constituída por 24 empresas, o que representa 85,3\% do total de GEFN na pesquisa.

${ }^{8}$ Os valores dispendidos na aquisição de outros conhecimentos externos não puderam ser divulgados devido às regras de sigilo do IBGE.

9 Ainda que baixo em relação ao padrão internacional do setor, esse montante é bastante significativo para a realidade brasileira (PARANHOS et al., 2016).
} 
de atividades inovativas, excluídas as atividades de P\&D interna, é feito 85,3\% pelas próprias empresas e 14,7\% por financiamento público. Entre as 22 empresas que receberam financiamento do governo, 29,2\% foram para projetos de P\&D com universidades.

A pouca relevância dada às atividades de parcerias é reforçada quando analisadas as fontes de informação e os parceiros na cooperação. Somente $34,7 \%$ das GEFN identificaram as universidades como fontes de informação com alto grau de importância. Os institutos de pesquisa, no entanto, destacam-se um pouco mais, pois foram identificados como de alta importância por 48,2\% das empresas na Pintec de 2014. A interação se dá majoritariamente com ICTs no Brasil, com apenas uma empresa declarando ter uma universidade no exterior como fonte de informação, e duas empresas indicando institutos de pesquisa localizados no exterior.

Os dados da cooperação efetiva divergem dos dados das fontes de informação, pois somente oito empresas declararam alto grau de importância na cooperação efetiva com universidades e institutos de pesquisa. Ou seja, ainda que sejam utilizados com fontes de informação, não necessariamente é estabelecida cooperação formal relevante para o processo inovativo das empresas. Entre as empresas que cooperam (17), 49,5\% identificam as ICTs como de alta importância, pouco menos que os fornecedores, identificados por 50,6\% das GEFNs inovadoras que cooperam como de alta importância. Três empresas indicaram cooperação com ICTs no exterior, e 11 com ICTs no Brasil, mantendo o padrão de uma maior interação com instituições nacionais. A P\&D e os ensaios para testes de produtos são as principais atividades realizadas em cooperação com as ICTs, declaradas por $94 \%$ das empresas, enquanto 37,6\% das empresas indicaram outras atividades de cooperação. Aproveitando a contribuição de Paranhos, Mercadante e Hasenclever (2016), é importante destacar que o número de empresas cooperando com ICTs e que as identificam como um parceiro de alta importância para a inovação é crescente nas três últimas edições da Pintec.

\subsection{Cenário das grandes empresas farmacêuticas na pesquisa de campo em 2017}

Os dados mais recentes das pesquisas de inovação no Brasil mostram mudanças positivas nos esforços inovativos das GEFNs, tendência esta que envolve a aproximação com ICTs, conforme identificado na subseção 2.1. Nesta subseção, são apresentados os resultados da pesquisa de campo com seis GEFNs que buscam caracterizar e atualizar o cenário das parcerias com as ICTs dessas empresas que se destacam no setor farmacêutico brasileiro. Todas as seis são produtoras de medicamentos finais, sendo que duas possuem estrutura farmoquímica, quarto atuam no segmento de genéricos, e todas no segmento de similares, ou genéricos de marca. Todas estão envolvidas, individualmente ou por participação em joint venture, nas parcerias para desenvolvimento produtivo (PDP) para transferência de tecnologia e produção de biossimilares no Brasil ${ }^{10}$. Tratam-se, dessa maneira, de importantes empresas do mercado brasileiro com porte e recursos para uma atuação mais inovadora. São, assim, exemplos significativos de como o setor farmacêutico brasileiro está se organizando para geração de inovação e promoção das parcerias empresa-ICT.

Uma percepção geral a partir das entrevistas é que as seis GEFNs entendem hoje que o estabelecimento de uma estratégia realmente inovativa é essencial para sua sobrevivência no mercado farmacêutico brasileiro. Tais empresas passaram por um processo de modernização e

${ }^{10}$ Para maiores detalhes ver Gomes (2014) e Torres (2015). 
crescimento significativo nas duas últimas décadas, ganhando posições significativas no mercado e, com isso, recursos para novas trajetórias. Foi possível identificar algumas estratégias diferenciadas de organização para inovação. Em três casos, houve a separação de divisões específicas dentro das empresas para inovação radical e incremental, ou busca de novas moléculas e desenvolvimento de genéricos e/ou similares. $\mathrm{O}$ objetivo da divisão de inovação radical é a ampliação dos investimentos em uma trajetória inovativa de mais longo prazo, maior incerteza e maior potencial de retorno. Nesse caso, as empresas também fazem busca internacional, mas não têm estruturas de $\mathrm{P} \& \mathrm{D}$ no exterior.

Em outros três casos, as empresas estabeleceram comitês científicos internos como estratégia de identificação, seleção e implementação de projetos de inovação e de parceria com ICTs. No primeiro caso, o comitê é formado por pesquisadores renomados de universidades, institutos de pesquisa e da própria empresa. Conforme um entrevistado, o comitê busca ampliar a confiança entre pesquisadores e empresa: "Eu vou colocar cientista falando com cientista para que [desmistifique] esse problema que havia de a Academia ter medo de apresentar projetos para a indústria farmacêutica" (EFN8_2017). O comitê encontra-se periodicamente para seleção e acompanhamento dos projetos. Foi relatado que os pesquisadores externos são importantes fontes de recebimento de projetos de ICTs na empresa, pois são procurados por colegas de várias partes do Brasil. A equipe de cada comitê avaliará a pertinência e potencial do projeto para posterior encaminhamento aos trâmites de estabelecimento da parceria com o pesquisador e a ICT. Essa empresa também fez recentemente uma divisão de áreas de inovação radical e incremental e outra de genéricos. Os projetos encaminhados e avaliados pelo comitê são focados em inovação radical.

No segundo caso, é formado um comitê com membros externos com formação técnica e especialidade na área de cada projeto de inovação radical que a empresa inicia. A empresa não estabelece um comitê fixo devido à variedade de temas que precisam ser avaliados. Segundo o representante da empresa:

\begin{abstract}
Nós não temos um comitê cientifico fixo, nós montamos comitês [...] de acordo com a molécula que [estamos] estudando. [...] Por que ter um comitê cientifico para temas tão específicos? Uma hora nós estamos falando de saúde da mulher, outra hora nós estamos falando de transplantes [...]. Nós precisamos ter as melhores cabeças dentro dessas especialidades [...]. Então, desenhamos um protocolo, nós vamos falar com os especialistas para construir o protocolo. Nós trabalhamos com médicos assessores, para os temas do dia a dia, e quando nós temos esses temas, assim mais de projetos de pesquisa, aí nós montamos o nosso [comitê] técnico daquela especialidade (EFN10_2017).
\end{abstract}

No terceiro caso, a empresa possui um comitê científico formado por médicos renomados com relacionamento com universidades e com membros internos à empresa, que se reúnem mensalmente. Atualmente, a empresa tem dois comitês, em oncologia e geriatria, e os pesquisadores externos também são importantes fontes de novidades ou projetos para serem discutidos. Dois conceitos já foram aprovados pelo comitê e um deles está em fase de escalonamento.

A estratégia de internacionalização é realizada por duas empresas. A primeira empresa conjuga duas estratégias, comitê científico e internacionalização, que foi realizada por meio da compra de uma empresa especializada em P\&D para busca de parceiros no exterior. A segunda empresa adquiriu um centro de $P \& D$ para identificação e desenvolvimento de pesquisas no exterior e posterior desenvolvimento no Brasil. Em ambos os casos, as empresas buscaram estratégias de internacionalização para acessar novos conhecimentos no exterior. O Quadro 1 sistematiza as informações das estratégias das seis empresas entrevistadas. 
Quadro 1: Estratégias de inovação e interação com ICTs das GEFNs

\begin{tabular}{|l|l|c|}
\hline \multicolumn{1}{|c|}{ Estratégia } & \multicolumn{1}{|c|}{ Objetivo } & N. Empresas \\
\hline $\begin{array}{l}\text { Divisões específicas para } \\
\text { inovação radical e incremental }\end{array}$ & $\begin{array}{l}\text { Ampliação dos investimentos em uma trajetória } \\
\text { inovativa de mais longo prazo, maior incerteza e maior } \\
\text { potencial de retorno }\end{array}$ & 3 \\
\hline Comitês científicos internos & $\begin{array}{l}\text { Identificação, seleção e implementação de projetos de } \\
\text { inovação e de parceria com ICTs }\end{array}$ & 3 \\
\hline Internacionalização & $\begin{array}{l}\text { Busca de parceiros no exterior; Identificação de } \\
\text { pesquisas no exterior e posterior desenvolvimento no } \\
\text { Brasil }\end{array}$ & 2 \\
\hline
\end{tabular}

Fonte: Elaboração a partir da pesquisa de campo.

Nota: A soma do número de empresas é maior que seis, pois duas empresas possuem duas estratégias combinadas.

Nas parcerias com ICTs, especificamente, as seis empresas também se diferenciam de acordo com suas estruturas inovativas. Fato presente em todos os casos, porém, é a existência de parcerias para prestação de serviços, realização de ensaios para testes de produtos, conforme identificado na subseção 2.1. Pelo menos três empresas têm experiência de produtos que estão no mercado que contaram com a parceria de ICTs, seja na prestação de serviço, seja na pesquisa conjunta. As empresas com novas divisões separadas para inovação radical estão na busca por parceiros, identificando pesquisadores e grupos de pesquisa com potencial para parcerias científicas, codesenvolvimento e licenciamento. Uma das empresas, que apresenta parcerias efetivas mais fortemente nos estudos clínicos e na prestação de serviços, destacou o papel das universidades na formação de recursos humanos qualificados para atuar na empresa. Outra empresa está ampliando suas competências internas para interagir com ICTs e inovar a partir da criação de uma empresa no exterior. Pelo menos duas empresas declararam ter parcerias com universidades no exterior.

Em relação aos benefícios identificados nas parcerias com ICTs, as empresas relatam ser mais importante a ampliação das capacidades internas das empresas a partir do conhecimento externo, do que a possibilidade de redução de custos ou maior agilidade para responder a mudanças de mercado. Um dos entrevistados relatou o caso de um produto que só foi desenvolvido devido à parceria com uma ICT:

Por exemplo, [esse produto], a ideia foi nossa, mas foi graças ao trabalho feito na [Universidade] com o professor [...], que nós conseguimos desenvolver as bases do produto para que ele pudesse se transformar no produto que foi para a pesquisa clínica. Nós estamos agora em fase de registro. É uma associação de duas drogas muito antigas que, por um acaso, quando juntas, deram uma ação totalmente nova, que ninguém esperava (EFN7_2017).

O conhecimento buscado na ICT é principalmente relacionado à transferência de tecnologia, realização de testes e/ou consultoria para desenvolvimento de novos produtos e/ou processos, pesquisas complementares, que a empresa não pode realizar, e informações sobre tendências de P\&D. Com menor importância, segundo os entrevistados, o conhecimento presente nas ICTs para treinamento dos funcionários também é utilizado, assim como, o acesso à recémformados e pesquisadores para recrutamento. Ressalta-se, porém, o comentário do representante de uma das empresas que disse fazer a formação do profissional internamente, pois as graduações deixariam a desejar por serem muito atrasadas: 
A gente tem faculdades de formação da graduação muito ruins. Aí a situação é oposta. Eu tenho um programa de estagiários, que eu quero inclusive aumentar porque quase que aproveito $100 \%$, para farmacêuticos e químicos, e a gente praticamente forma o cara aqui dentro (EFN1_2017).

Vale notar ainda que, em geral, as empresas não utilizam as parcerias para acessar conhecimento para problemas da produção ou de controle de qualidade.

Em síntese, pode-se perceber pelas entrevistas que é possível diferenciar estratégias mais inovativas e de maior complexidade nas parcerias com ICTs quando analisadas de forma mais profunda empresas específicas que se destacam no setor. Os resultados ainda são incipientes, mas já é possível identificar estruturas e formas de atuação diferenciadas em relação ao setor farmacêutico brasileiro.

\section{Discussão e Conclusão}

A partir dos dados secundários da Pintec 2014, foi possível estabelecer um panorama geral das GEFNs sobre seus esforços e desempenhos inovativos. Como característica geral, elas inovam sozinhas utilizando majoritariamente recursos próprios. Menos da metade das empresas indicou aquisição externa de $\mathrm{P} \& \mathrm{D}$ ou outros conhecimentos. Em especial, o valor do dispêndio em aquisição de $\mathrm{P} \& \mathrm{D}$ externa foi menos de um quinto das atividades de $\mathrm{P} \& \mathrm{D}$ interna. Essas fontes externas de informação são principalmente ICTs nacionais, com destaque para os institutos de pesquisa. No entanto, ainda é baixa a relevância dessas ICTs para os esforços de cooperação efetiva das GEFNs, apesar de essa interação ter crescido nos últimos anos.

No entanto, em uma perspectiva menor com enfoque em seis GEFNs com grande destaque no mercado nacional, na utilização dos instrumentos de financiamento disponíveis ao setor, participação nas PDPs e em parcerias, foi possível a identificação de estratégias diferenciadas e mais significativas de busca inovativa e interação com ICTs. Resumidamente, as estratégias de criação de departamentos de inovação radical, comitês científicos e internacionalização mostram-se acertadas e de acordo com a literatura de gestão da inovação.

Adotar uma estratégia de inovação mais avançada é importante para a sobrevivência das empresas oriundas de indústrias intensivas em tecnologia. Como visto na pesquisa de campo, após passarem por um período de altas taxas de crescimento, as GEFNs estão direcionando esforços para aprimorar as suas capacidades inovativas, sendo que uma das formas é a implantação de um departamento de inovação radical. McDermott e O'Connor (2002) evidenciaram que investir em inovação radical é um elemento crítico para o sucesso de longo prazo das empresas. A gestão da inovação radical deve ser feita à parte da gestão da inovação incremental devido aos diferentes níveis de complexidade e incerteza (DEWAR; DUTTON, 1986) e diferentes formas de criação de capacidade de absorção do conhecimento (FORÉS; CAMISÓM, 2016). Neste sentido, parece acertada a nova estrutura estabelecida nas GEFNs de separação entre os departamentos de inovação radical, incremental e genérico. E é valido de nota que a estrutura financeira e de recursos humanos de uma grande empresa é um importante fator de viabilidade desta estratégia.

Tal estratégia combinada, em determinados casos, aos comitês científicos internos e/ou à atuação internacional na busca por parceiros externos, tendem a potencializar a possibilidade de agregar conhecimentos externos ao conhecimento interno da empresa para geração de inovação. No contexto dos países em desenvolvimento, estudos (ALBUQUERQUE, 1996; 
KATZ, 2000; VELHO; SAENZ, 2002) apontam que as empresas possuem capacidade tecnológica limitada, que dificulta a conexão com ICTs. Na maioria dos casos em que ocorre a interação, ela é realizada para substituir os esforços internos de inovação, e não complementares. Esta realidade ainda é bem presente no caso do setor farmacêutico brasileiro, mas conforme os dados secundários, as GEFNs estão ampliando seus esforços em P\&D interna para ampliar suas capacidades de absorção de conhecimentos externos. Os resultados da Pintec e da pesquisa de campo demonstram que há empresas no setor farmacêutico brasileiro com maiores tendências ao desenvolvimento inovativo, ainda que não eliminadas as limitações de empresas de países em desenvolvimento dependentes tecnologicamente.

Como meio de ultrapassar tal limitação, uma estratégia de inovação cada vez mais usada por empresas de diversos setores é a internacionalização da $\mathrm{P} \& \mathrm{D}$, porque o conhecimento se dispersa além das fronteiras nacionais. Não obstante, o conhecimento científico específico para determinados setores é concentrado por alguns países, especialmente os desenvolvidos. Esses fatores motivam as empresas a buscarem acessar o conhecimento externo estrangeiro, como foi identificado nos dados da Pintec e na pesquisa de campo. A internacionalização da P\&D é uma estratégia de gestão da inovação frequentemente usada pelas grandes empresas farmacêuticas, conforme apontam Zedtwitz e Gassmann (2002). A maioria dessas empresas possuem redes integradas de $\mathrm{P} \& \mathrm{D}$, de modo que a pesquisa está localizada onde há informações científicas de alta qualidade providas por centros de excelência. A gestão da P\&D no ambiente internacional é mais complexa, porém os custos adicionais de manter tais atividades são compensados pela criação de novos negócios e ganhos de vantagens competitivas. Participar de redes globais de P\&D faz com que o conhecimento seja mais rapidamente absorvido e adaptado para outros lugares, ganhando eficiência no lançamento de produtos no mercado. Os resultados do estudo apresentado neste artigo mostram que as GEFN também estão seguindo esta tendência do setor.

O estudo apresentado neste artigo não pode ser generalizado para o setor farmacêutico brasileiro por se tratar de um estudo de caso com seis GEFNs. Além disso, ainda é cedo para concluir sobre o sucesso das estratégias identificadas. No entanto, é válido ressaltar o diferencial das estratégias dessas empresas frente à média do setor e da indústria brasileira como um todo e as contribuições que estes resultados trazem para a literatura da relação empresa-ICT no Brasil. Neste sentido, é possível destacar que: a) em setores intensivos em tecnologia que requerem altos investimentos, o porte das empresas é significativo para o desenvolvimento da inovação, seja por maior capacidade financeira e de pessoal, seja por sua maior habilidade política para acessar os instrumentos de estímulo do governo; b) apesar da característica de empresas de países dependentes tecnologicamente que utilizam ICTs de forma substitutiva, já é possível encontrar estratégias, ainda que incipientes, de utilização das ICTs de forma complementar aos esforços internos de P\&D das empresas; c) há empresas que montaram estruturas capazes de sobrepor as limitações de divulgação de suas pesquisas e resultados tecnológicos presentes no sistema científico e tecnológico brasileiro, que permitem a identificação destes novos conhecimento; d) as empresas começam a entender que estratégias focadas somente no mercado local são limitadoras e iniciam estratégias, ainda que tímidas, de busca de novos conhecimentos no exterior. Todas as estratégias ainda são incipientes e presentes em poucas empresas do setor, neste sentido, é válida a realização de estudos futuros para o acompanhamento da atuação das GEFNs no Brasil. 


\section{Referências}

ALBUQUERQUE, E. Sistema nacional de inovação no Brasil: uma análise introdutória a partir de dados disponíveis sobre a ciência e a tecnologia. Revista de Economia Política, 16(3), 1996, 56-72.

ARORA, A.; GAMBARDELLA, A. Complementarity and External Linkages: The Strategies of the Large Firms in Biotechnology. The Journal of Industrial Economics, [s.1.], v. 38, n. 4, p.361-379, jun. 1990.

BELDERBOS, R. et al. Heterogeneity in R\&D cooperation strategies. International Journal of Industrial Organization, [s.1.], v. 22, n. 8-9, p.1237-1263, nov. 2004.

BERCOVITZ, J.; FELDMAN, M. Fishing upstream: Firm innovation strategy and university research alliances. Research Policy, [s.1.], v. 36, n. 7, p.930-948, set. 2007.

BLAKESLEE, W.; Licensing, partnering, strategic alliances and university relationships. Journal of Commercial Biotechnology, v. 18, p. 68-71, 2012.

CASTRO, P.; TEIXEIRA, A.; LIMA, J. A relação entre os canais de transferência de conhecimento das Universidades/IPPS e o desempenho inovativo das firmas no Brasil. Revista Brasileira de Inovação, [s.1.], v. 13, n. 2, p.345-370, 1 abr. 2014.

CGEE - CENTRO DE GESTÃO DE ESTUDOS ESTRATÉGICOS. Competências para inovar na indústria farmacêutica brasileira. Brasília: Centro de Gestão e Estudos Estratégicos, 2017.

CHAVES, C. et al. The point of view of firms in Minas Gerais about the contribution of universities and research institutes to R\&D activities. Research Policy, n. 41, p. 1683- 1695, 2012.

CHAVES, G.; HASENCLEVER, L.; OLIVEIRA, M. Conexões entre as políticas de desenvolvimento industrial no setor farmacêutico e a política de saúde no Brasil: um percurso da década de 1930 a 2000. In: HASENCLEVER, L.; OLIVEIRA, M.; PARANHOS, J.; CHAVES, G. (Org.) Desafios de operação e desenvolvimento do complexo industrial da saúde. Rio de Janeiro: E-papers, 2016.

COHEN, W.; LEVINTHAL, D. Absorptive capacity: a new perspective on learning and innovation. Administrative Science Quarterly, v. 35, n. 1, Special issue: Technology, Organizations and Innovation, p. 128-152, 1990.

COHEN, W.; NELSON, R.; WALSH, J. Links and Impacts: The Influence of Public Research on Industrial R\&D. Management Science, [s.1.], v. 48, n. 1, p.1-23, jan. 2002.

DELGADO, I. Política industrial para os setores farmacêutico, automotivo e têxtil na China, Índia e Brasil. Brasília: IPEA, Texto de Discussão, n. 2087, 2015.

DEL-VECCHIO, R.; BRITTO, J.; OLIVEIRA, B. Patterns of university-industry interactions in Brazil: an exploratory analysis using the instrumental of graph theory. Quality \& Quantity, [s.1.], p.1-26, 7 maio 2013.

DEWAR, R.; DUTTON, J. The Adoption of Radical and Incremental Innovations: An Empirical Analysis. Management Science, [s.1.], v. 32, n. 11, p.1422-1433, nov. 1986.

DODGSON, M. Technological Collaboration in Industry: Strategy, Policy, and Internationalization in Innovation. Routledge, 1993. 
FILIERI, R. et al. Structural social capital evolution and knowledge transfer: Evidence from an Irish pharmaceutical network. Industrial Marketing Management, [s.1.], v. 43, n. 3, p.429440, abr. 2014.

FORÉS, B.; CAMISÓN, C. Does incremental and radical innovation performance depend on different types of knowledge accumulation capabilities and organizational size? Journal of Business Research, [s.1.], v. 69, n. 2, p.831-848, fev. 2016.

GOMES, E. B. P. Clusters e Biotecnologia para a superação da imitação: estudo de caso da indústria farmacêutica brasileira. Rio de Janeiro: Programa de Pós-graduação em Políticas Públicas, Estratégias e Desenvolvimento/Instituto de Economia/UFRJ, 2014. (Tese de Doutorado)

HASENCLEVER, L et al. Uma análise das políticas industriais e tecnológicas entre 20032014 e suas implicações para o complexo industrial da saúde. In: HASENCLEVER, L.; OLIVEIRA, M.; PARANHOS, J.; CHAVES, G. (Org.) Desafios de operação e desenvolvimento do complexo industrial da saúde. Rio de Janeiro: E-papers, 2016.

HESS, A.; ROTHAERMEL, F. When Are Assets Complementary? Star Scientists, Strategic Alliances, and Innovation in the Pharmaceutical Industry. Strategic Management Journal, v. 32, p. 895-909, 2011.

HOTTENROTT, H.; LOPES-BENTO, C. Quantity or Quality? Collaboration Strategies in Research and Development and Incentives to Patent. ZEW Discussion Paper, n. 47, 2012.

KATZ, J. Pasado y presente del comportamiento tecnológico de América Latina. 75. ed. Santiago: Cepal, 2000. 80 p. (Series de la CEPAL).

LEMOS, D.; CARIO, S. Análise da interação universidade-empresa para o desenvolvimento inovativo a partir da perspectiva teórica institucionalista-evolucionária. Revista Brasileira de Inovação, [s.1.], v. 14, n. 2, p.361-382, 10 set. 2015.

MARCONI, M. A.; LAKATOS, E. M. Fundamentos de metodologia científica. $7^{\text {a }}$. ed. São Paulo: Atlas, 2016.

MCDERMOTT, C.; O'CONNOR, G. Managing Radical Innovation: An Overview of Emergent Strategy Issues. Journal of Product Innovation Management. 19. 424-438, 2002.

PARANHOS, J. Interação entre empresas e instituições de ciência e tecnologia: o caso do sistema farmacêutico de inovação brasileiro. Rio de Janeiro: EdUERJ, 2012.

PARANHOS, J.; MERCADANTE, E. O panorama político-econômico da harmonização ao Acordo Trips e da consolidação do setor farmacêutico brasileiro. In: MEZENES, H. (Org.) Propriedade Intelectual, Inovação Tecnológica e Saúde. João Pessoa: Editora UFPB, 2017.

PARANHOS, J.; MERCADANTE, E.; HASENCLEVER, L. Alteração no padrão de esforços de inovação das grandes empresas farmacêuticas no Brasil, 2008-2011. In: HASENCLEVER, L.; OLIVEIRA, M.; PARANHOS, J.; CHAVES, G. (Org.) Desafios de operação e desenvolvimento do complexo industrial da saúde. Rio de Janeiro: E-papers, 2016.

PARANHOS, J. et al. Potenciais e vulnerabilidades da interação empresa e instituições científicas e tecnológicas no setor farmacêutico: a visão dos atores. In: HASENCLEVER, L.; PARANHOS, J.; CHAVES, G.; OLIVEIRA, M. (Org.) Vulnerabilidades do Complexo Industrial da Saúde: reflexos das políticas industrial e tecnológica na produção local e assistência farmacêutica. Rio de Janeiro: E-papers, 2018. 
PAVITT, K. Sectoral patterns of technical change: Towards a taxonomy and a theory. Research Policy, [s.1.], v.13, n. 6, p.343-373, dez. 1984.

PIMENTEL, V. P. Apropriação da Inovação na Indústria Farmacêutica: Um novo modelo de negócios? 2010. 78 f. Monografia - Curso de Ciências Econômicas, Instituto de Economia, Universidade Federal do Rio de Janeiro, Rio de Janeiro, 2010.

PISANO, G. The governance of innovation: Vertical integration and collaborative arrangements in the biotechnology industry. Research Policy, [s.1.], v. 20, n. 3, p.237-249, jun. 1991.

RAPINI, M.; OLIVEIRA, V.; CALIARI, T. Como a interação universidade-empresa é remunerada no Brasil: evidências dos grupos de pesquisa do CNPq. Revista Brasileira de Inovação, [s.1.], v. 15, n. 2, p.219-246, 28 ago. 2016.

RAPINI, M.. et al. University-industry interactions in an immature system of innovation: evidence from Minas Gerais, Brazil. Science and Public Policy 36, p. 373-386, 2009.

RADAELLI, V. A Nova Conformação Setorial da Indústria Farmacêutica Mundial: redesenho nas pesquisas e ingresso de novos atores. Revista Brasileira de Inovação, [s.1.], v. 7, n. 2, p.445-482, 24 jun. 2008.

ROSENBERG, N.; NELSON, R. American universities and technical advance in industry. Research Policy, [s.1.], v. 23, n. 3, p.323-348, may, 1994.

SCHILLING, M. Strategic Management of Technological Innovation. New York: McGrawHill Irwin, 2006.

SOH, P-H.; SUBRAMANIAN, A. When do firms benefit from university-industry R\&D collaborations? The implications of firm R\&D focus on scientific research and technological recombination. Journal of Business Venturing, v. 29, p. 807-821, 2014.

STRÜCKER, A.; CYTRYNOWICZ, M. Origens e trajetórias da indústria farmacêutica no Brasil. São Paulo: Narrativa Um, 2007.

STUART, T.E. (2000). Interorganizational Alliances and the Performance of Firms: A Study of Growth and Innovation Rates in a Hightechnology Industry. Strategic Management Journal 21: 791-811, 2000.

SUZIGAN, W.; ALBUQUERQUE, E. The underestimated role of universities for the Brazilian system of innovation. Revista de Economia Política, [s.1.], v. 31, n. 1, p.03-30, mar. 2011.

TEECE, D. Profiting from Technological Innovation: implications for integration, collaboration, licensing and public policy. In: TEECE, D. (org.) The Competitive Challenge: strategies for industrial innovation and renewal. Cambridge: Balinger Publishing Company, 1987.

TEECE, D. Explicating Dynamics Capabilities: the nature and microfoundations of (sustainable) enterprise performance. Strategic Management Journal, v. 28, p. 1319-1350, 2007.

TEIXEIRA, A. et al. Dimensões da capacidade de absorção, qualificação da mão de obra, P\&D e desempenho inovativo. Revista Brasileira de Inovação, [s.1.], v. 15, n. 1, p.139-163, 16 mar. 2016. 
TORRES, R. L. Capacitação Tecnológica na Indústria Farmacêutica Brasileira. Rio de Janeiro: Programa de Pós-graduação em Economia/Instituto de Economia/UFRJ, 2015. (Tese de Doutorado)

TRALAU-STEWART, C. J. et al. Drug discovery: new models for industry-academic partnerships. Drug Discovery Today, [s.1.], v. 14, n. 1-2, p.95-101, jan. 2009.

TRIULZI, G.; PYKA, A.; SCHOLZ, R. R\&D and knowledge dynamics in university-industry relationships in biotech and pharmaceuticals: an agent-based model. Int. J. Biotechnology, v. 13, n. 1/2/3, p. 137-179, 2014.

VELHO, L.; SAENZ, T. R\&D in the public and private sector in Brazil: complements or substitutes? Discussion Paper 2002-8 UNU/INTECH. Acessado em: 15/01/2003. Disponível em: http://www.intech.unu.edu/publications/discussion-papers/2002-8.pdf

VEUGELERS, R.; CASSIMAN, B. R\&D cooperation between firms and universities. Some empirical evidence from Belgian manufacturing. International Journal of Industrial Organization, [s.1.], v. 23, n. 5-6, p.355-379, jun. 2005.

ZEDTWITZ, M.; GASSMANN, O. Market versus technology drive in R\&D internationalization: four different patterns of managing research and development. Research Policy, [s.1.], v. 31, n. 4, p.569-588, may 2002.

YIN, R. Estudo de caso: planejamento e métodos. $5^{\text {a }}$ edição. Porto Alegre: Bookman, 2015. 